\title{
Influência do Uso do Smartphone Sobre o Equilíbrio de Jovens Durante a Realização de Dupla-Tarefa
}

\author{
Influence of Smartphone Use on the Balance of Young Adults During Dual Task
}

\section{RESUMO}

Objetivo: Avaliar o impacto do uso de smartphones sobre o equilíbrio estático e dinâmico de adultos jovens e calcular a amostra ideal a partir do tamanho do efeito obtido nesse estudo. Metodologia: Dez adultos jovens com idade de 21,5 $\pm 3,2$ anos foram incluídos nesse estudo. Os participantes foram submetidos a testes estáticos (plataforma de força) e dinâmicos (teste Timed Get Up and Go), realizados concomitantes ao uso do smartphone. A análise estatística envolveu os testes de Friedmann e Wilcoxon, comparando os resultados com e sem o uso de celular. Resultados: O uso de smartphone impactou negativamente o equilíbrio estático e dinâmico dos participantes, tanto em atividades de digitação de mensagem quanto em atividades de conversa ao telefone. A análise do tamanho do efeito sugere a necessidade de 45 jovens para mensuração do equilíbrio estático e 28 para análise do equilíbrio dinâmico. Conclusão: O uso do smartphone causou interferência sobre o equilíbrio de adultos jovens, predispondo a desequilíbrios e riscos de quedas em atividades estáticas e dinâmicas. Os resultados devem ser corroborados por novos estudos com tamanho amostral superior a 45 participantes.

\section{DESCRITORES}

Equilibrio Postural. Acidentes por Quedas. Telefone Celular. Viés de Atenção. Atenção à Saúde.

\begin{abstract}
Objective: The use of smartphones has become increasingly common, especially among young adults. The aim of this study was to assess the impact of the use of smartphones on the static and dynamic balance in young adults and to calculate the ideal sample from the effect size seen in this study. Methodology: Ten young adults with mean age of $21.5 \pm 3.2$ years were enrolled in this study. The participants underwent static balance (force platform) and dynamic (Timed Get Up and Go) tests, performed concurrently when in use of a smartphone. The statistical analysis was assessed with the Friedman and Wilcoxon tests, comparing the results with and without the use of cell phone. The effect size related to the interference of the use of smartphone was assessed on static and dynamic balance. Significance was set at $5 \%$. Results: The use of smartphone impacted the static and dynamic balance of the participants, in both activities of typing messages or speaking on the phone. Effect size analysis suggests the need for 45 young adults to measure static balance and 28 young adults to assess dynamic balance. Conclusion: The use of smartphone caused interference on the balance of young adults, predisposing to imbalances and risks of falls in static and dynamic tasks. The results must be corroborated by new studies with sample sizes greater than 45 participants.
\end{abstract}

\section{DESCRIPTORS}

Postural Balance. Accidental Falls. Cell Phone. Attentional Bias. Health Care.

${ }^{1}$ Aluno do Instituto Integrado de Saúde da Universidade Federal de Mato Grosso do Sul. Campo Grande, Mato Grosso do Sul, Brasil.

${ }^{2}$ Aluno do Curso de Mestrado do Programa de Pós-Graduação em Ciências do Movimento da Universidade Federal de Mato Grosso do Sul. Campo Grande, Mato Grosso do Sul, Brasil.

${ }^{3}$ Professor doutor e orientador do Programa de Pós-graduação em Ciências do Movimento da Universidade Federal de Mato Grosso do Sul. Campo Grande, Mato Grosso do Sul, Brasil.

${ }^{4}$ Professor doutor e orientador do Programa de Pós-Graduação em Saúde e Desenvolvimento da Universidade Federal de Mato Grosso do Sul. Campo Grande, Mato Grosso do Sul, Brasil. 
mundo vem observando nos últimos anos uma proliferação de telefones multiusos, classificados como smartphones. A função de um smartphone está muito além do que ligar e receber chamadas. Hoje em dia, o telefone celular permite acesso rápido à internet, possibilita conversas em redes sociais, apresenta recursos de tirar e divulgar fotos, fazer chamadas de vídeo e dá outras funções provenientes de aplicativos com possibilidades educativas diversas ${ }^{1,2}$.

$\mathrm{O}$ avanço tecnológico tem ampliado o acesso virtual de jovens e tornado a comunicação digital um meio importante de troca de informações ${ }^{3,4}$. Nesse aspecto, nota-se cada vez mais o aumento da demanda cognitiva atencional no dia a dia dos jovens, visto que é comum ver as pessoas caminhando e manuseando o celular, fazendo atividades na academia e se distraindo com músicas e jogos e utilizando o celular ao mesmo tempo em que faz as refeições ${ }^{5}$. De um lado esse recurso permite a utilização da reserva cognitiva do indivíduo para dar conta de atividades concomitantes, mas de outro a atenção dividida em multitarefas pode possibilitar maior risco frente a distração nas tarefas realizadas ${ }^{6}$.

A realização de tarefas duplas ocorre quando o indivíduo se propõe a realizar uma atividade e ao mesmo tempo é distraído por uma outra demanda. Com o aperfeiçoamento dos recursos dos celulares, estudos demonstram o quão os aparelhos smartphones vêm impactando a vida das pessoas, com possíveis riscos de realização de tarefas concomitantes e a consequente divisão da atenção entre elas ${ }^{5-8}$.

No Brasil, dados do Instituto Brasileiro de Geografia e Estatística (IBGE) ${ }^{9}$ reve- lam que o smartphone é o principal meio de comunicação virtual entre jovens, deixando os computadores, os tablets e os notebooks em segundo plano, em $98 \%$ dos acessos. O IBGE ${ }^{9}$ também aponta que o acesso à internet no país alcançou 126,4 milhões de usuários, ocorrendo o maior acesso na faixa etária entre 18 e 24 anos. No cenário do uso crescente de smartphones no dia a dia das pessoas, realizou-se esse estudo que teve como objetivos avaliar o impacto do uso de smartphones sobre o equilíbrio estático e dinâmico de adultos jovens e realizar o cálculo amostral ideal, a partir do tamanho do efeito da interferência do uso do smartphone sobre o equilíbrio dos participantes. A hipótese dos pesquisadores era a de que a realização de tarefas motoras concomitantes ao uso de celular causariam alterações do equilíbrio estático e dinâmico, aumentando o risco de quedas dos participantes.

\section{METODOLOGIA}

Sob um delineamento transversal e com análises quantitativas de dados, 10 adultos jovens, com idade de $21,5 \pm 3,2$ anos, participaram desse estudo piloto. Os participantes foram recrutados em Campo Grande/MS e todos os sujeitos forneceram consentimento por escrito antes das avaliações. A pesquisa foi realizada de acordo com a Declaração de Helsinki e a Resolução n. 466/2012, do Conselho Nacional de Saúde (CNS), após a aprovação do projeto pelo Comitê de Ética em Pesquisa da Universidade Federal de Mato Grosso do Sul.

Como critérios de inclusão foram admitidos adultos jovens que se encontravam 
na faixa etária dos 18 aos 25 anos, de ambos os sexos e que não apresentavam disfunções de origem ósseo-mio-articular, neurológica ou psiquiátrica. Foram excluídas pessoas que faziam uso de medicação contínua, aquelas que relataram episódio prévio de labirintite e as que não dispunham de possibilidade para comparecer ao laboratório de biomecânica do Programa de Pós-graduação em Ciências do Movimento da Universidade Federal de Mato Grosso do Sul. Participantes que não tinham e que também nunca utilizaram smartphones foram excluídos da pesquisa.

\section{Procedimentos Metodológicos}

Todos os procedimentos metodológicos estão descritos de acordo com o STROBE checklist ${ }^{10}$. As variáveis desse estudo envolveram testes cognitivos, funcionais e de equilíbrio. A seguir encontra-se o detalhamento dos instrumentos utilizados.

A análise cognitiva se deu pelo Mini-Exame do Estado Mental (MEEM) ${ }^{11}$, pela Bateria de Avaliação Frontal (BAF) $)^{12}$ e pelo teste de fluência verbal semântica $(T F V)^{13}$. Enquanto o MEEM é responsável pela análise geral da cognição, a BAF e o TFV centram suas análises nas funções cognitivas pré-frontais. O objetivo da inclusão desses testes foi o de analisar diversos aspectos cognitivos dos participantes, antes desses serem submetidos a situações de duplas-tarefas com smartphone.

A funcionalidade dos participantes foi mensurada pelo Índice de Pfeffer ${ }^{14}$. Esse teste é importante, pois avalia a independência funcional dos participantes em atividades instrumentais da vida diária e possíveis alterações na funcionalidade podem afetar os resultados e causar vieses sobre a pesquisa.
O equilíbrio estático dos participantes foi analisado na plataforma de força BIOMEC 400_V4. Os participantes realizaram todos os testes com os pés descalços e foram instruídos a permanecer na plataforma de força por 60 segundos. Os participantes foram avaliados em situações com e sem uso de smartphones. Durante o uso de smartphones, os participantes realizaram duas atividades: $\left.1^{\circ}\right)$ Digitar a seguinte mensagem em seu smartphone: "Bom dia, estou no trabalho e vou chegar atrasado para nosso compromisso"; e $2^{\circ}$ ) Atender uma ligação telefônica realizada pelos pesquisadores, estando o telefone do participante em seu bolso dianteiro. A ordem de aplicação das diversas situações na plataforma de força foi aleatória, para evitar influência de aprendizagem. As seguintes variáveis foram incluídas na análise estabilométrica: deslocamento anteroposterior e médio-lateral $(\mathrm{cm})$, área de deslocamento $\left(\mathrm{cm}^{2}\right)$ e velocidade de deslocamento anteroposterior e médio-lateral $(\mathrm{cm} / \mathrm{s})$.

O equilíbrio dinâmico dos participantes foi mensurado pelo teste Timed Get Up and Go $(T U G)^{15}$. O teste é contabilizado em segundos e o número de passos mediante a realização da atividade de se levantar de uma cadeira, caminhar 3 metros, virar, voltar à cadeira e se sentar. De forma similar à plataforma de força, o teste foi realizado em condição simples e diante do uso de smartphones (mesmas atividades propostas na plataforma de força). A ordem de aplicação das diversas situações no TUG foi aleatória.

Análise Estatística

A análise dos dados envolveu a esta- 
tística descritiva e inferencial. Como os dados não cumpriram os pressupostos paramétricos de normalidade e homogeneidade de variâncias, foram utilizadas análises não-paramétricas tanto para a estatística descritiva (caracterizada como mediana e intervalo interquartil) quanto para a estatística inferencial.

Para a estatística inferencial, o teste Friedman associado ao pós-teste de Wilcoxon foi utilizado para análise do equilíbrio dos participantes com e sem o uso de smartphones. O tamanho do efeito do impacto do uso do smartphone sobre o equilíbrio foi calculado registrando o poder estatístico de cada comparação. Para todas as análises foi admitido um nível de significância de 5\%.

\section{RESULTADOS}

Dez pessoas, sendo 5 homens e 5 muIheres, participaram desse estudo. A Tabela 1 detalha as características gerais da amostra.

A Tabela 2 demonstra que o uso do smartphone afetou o equilíbrio estático dos participantes, sobretudo nas variáveis área e velocidade de deslocamento. Análises do pós-teste de Wilcoxon apontaram que o ato de digitar ao celular e permanecer em pé ocasiona um desequilíbrio maior na comparação da pessoa ficar em pé sem a atividade de dupla tarefa $(p=0,005$ para área, $p=0,015$ para velocidade anteroposterior e $p=0,012$ para velocidade médio-lateral). De forma similar, a atividade de falar ao telefone em pé ocasiona um maior desequilíbrio do que a atividade ficar em pé sem a atividade distratora ( $p=0,007$ para área, $p=0,005$ para velocidade anteroposterior e $p=0,005$ para velocidade médio-lateral). A comparação da atividade de digitar com a atividade de falar ao telefone demonstrou pior equilíbrio na atividade de falar ao telefone ( $p=0,028$ para área, $p=0,017$ para velocidade anteroposterior e $p=0,022$ para velocidade médio-lateral).

A Tabela 3 demonstra o impacto negativo que o uso do smartphone gera sobre o equilíbrio dinâmico dos participantes. Análises do pós-teste de Wilcoxon apontaram que o

Tabela 1. Aspectos sociais, cognitivos e funcionais dos participantes

\begin{tabular}{lc}
\hline \multicolumn{1}{c}{ Variáveis } & Participantes \\
Tamanho amostral & $\mathrm{n}=10$ \\
\hline Sexo (masculino:feminino) & $5: 5$ \\
& Média \pm DP \\
\hline Idade (anos)- & $21,5 \pm 3,2$ \\
Tempo de uso do smartphone (anos) & $11,0 \pm 4,2$ \\
Uso diário do smartphone (horas) & $4,0 \pm 2,0$ \\
Mini-Exame do Estado Mental (pontos) & $29,3 \pm 1,0$ \\
Bateria de Avaliação Frontal (pontos) & $17,0 \pm 1,5$ \\
Fluência verbal (animais/minuto) & $22,0 \pm 7,0$ \\
Índice de Pfeffer (pontos) & - \\
\hline Os dados estão expressos em número de eventos (para tamanho amostral e sexo) e mediana \pm in- \\
tervalo interquartil para demais variáveis.
\end{tabular}


Tabela 2. Impacto do uso do smartphone sobre as variáveis estabilométricas dos participantes

\begin{tabular}{lcccc}
\hline Variáveis & Tarefa simples & $\begin{array}{c}\text { Dupla-tarefa com } \\
\text { digitação }\end{array}$ & $\begin{array}{c}\text { Dupla-tarefa com } \\
\text { ligação }\end{array}$ & $p$ \\
\hline Deslocamento AP $(\mathrm{cm})$ & $-4,8 \pm 2,2$ & $-5,5 \pm 2,2$ & $-5,1 \pm 2,3$ & 0,497 \\
Deslocamento ML $(\mathrm{cm})$ & $-0,8 \pm 0,6$ & $-1,0 \pm 0,7$ & $-0,8 \pm 1,0$ & 0,497 \\
Área de deslocamento $\left(\mathrm{cm}^{2}\right)$ & $2,2 \pm 2,9$ & $4,1 \pm 3,2^{\mathrm{a}}$ & $7,6 \pm 10,3^{\mathrm{a}, \mathrm{b}}$ & 0,001 \\
Velocidade de deslocamento AP & $0,9 \pm 0,1$ & $1,1 \pm 0,1^{\mathrm{a}}$ & $1,4 \pm 0,7^{\mathrm{a}, \mathrm{b}}$ & 0,001 \\
$(\mathrm{~cm} / \mathrm{s})$ & $1,0 \pm 0,2$ & $1,1 \pm 0,3^{\mathrm{a}}$ & $1,4 \pm 0,3^{\mathrm{a}, \mathrm{b}}$ & 0,001 \\
$\begin{array}{l}\text { Velocidade de deslocamento ML } \\
(\mathrm{cm} / \mathrm{s})\end{array}$ & & & \\
\hline
\end{tabular}

Os dados estão expressos em mediana \pm intervalo interquartil para demais variáveis. Valor de $p$ do teste de Friedman. ${ }^{a}=$ diferença no mesmo grupo comparado com a atividade de tarefa simples. ${ }^{b}=$ diferença no mesmo grupo comparado com a atividade de dupla-tarefa com digitação.

Tabela 3. Impacto do uso do smartphone sobre o equilíbrio dinâmico dos participantes

\begin{tabular}{lcccc}
\hline Variáveis & Tarefa simples & $\begin{array}{c}\text { Dupla-tarefa com } \\
\text { digitação }\end{array}$ & $\begin{array}{c}\text { Dupla-tarefa com } \\
\text { ligação }\end{array}$ & $p$ \\
\hline Tempo (s) & $9,0 \pm 2,0$ & $11,0 \pm 1,0^{\mathrm{a}}$ & $10,5 \pm 2,2^{\mathrm{a}}$ & 0,001 \\
Passos $(\mathrm{n})$ & $14,0 \pm 2,2$ & $16,0 \pm 1,5^{\mathrm{a}}$ & $15,5 \pm 2,5^{\mathrm{a}}$ & 0,001 \\
\hline
\end{tabular}

Os dados estão expressos em mediana \pm intervalo interquartil para demais variáveis. Valor de $p$ do teste de Friedman. adiferença no mesmo grupo comparado com a atividade de tarefa simples.

Tabela 4. Cálculo do tamanho amostral levando-se em consideração análises multivariadas e univariadas

\begin{tabular}{lccc}
\hline \multicolumn{1}{c}{ Variáveis } & $\begin{array}{c}\text { Tamanho do } \\
\text { efeito }\end{array}$ & $\begin{array}{c}\text { Poder estatístico } \\
(\%)\end{array}$ & $\begin{array}{c}\text { Tamanho amostral } \\
\text { necessário }\end{array}$ \\
\cline { 1 - 1 } \cline { 3 - 4 } Deslocamento anteroposterior & 0,160 & 31,2 & 534 \\
Deslocamento médio-lateral & 0,067 & 14,1 & 3.034 \\
Área & 0,563 & 98,3 & 45 \\
Velocidade anteroposterior & 0,635 & 99,8 & 36 \\
Velocidade médio-lateral & 0,649 & 99,8 & 35 \\
TUG tempo & 0,730 & 99,9 & 28 \\
TUG passos & 0,798 & 99,9 & 24 \\
\hline
\end{tabular}

Cálculo do tamanho amostral realizado a partir do tamanho do efeito identificado nessa pesquisa, com delimitação do erro tipo 1 em $5 \%$ e poder estatístico em $95 \%$. 
ato de digitar e caminhar ocasiona um maior tempo $(p=0,004)$ e uma maior quantidade de passos $(p=0,004)$ para realizar a tarefa, na comparação do teste realizado sem o celular. De forma similar, falar ao telefone e caminhar repercute em maior tempo $(p=0,005)$ e número de passos $(p=0,007)$. Não ocorreu diferença significativa para o tempo $(p=0,527)$ e os passos ( $p=0,059)$, quando se compara as atividades de caminhada com digitação à atividade de caminhada com conversa ao telefone.

A Tabela 4 refere-se ao tamanho do efeito identificado nas análises pareadas, bem como o poder estatístico e o tamanho amostral ideal.

\section{DISCUSSÃO}

O objetivo desse estudo foi avaliar se o equilíbrio de adultos jovens sofre interferência quando da realização de atividades com o smartphone. Os resultados comprovaram que atividades de digitação de mensagem e falar ao telefone celular causam uma maior instabilidade motora, tanto em situações de ortostatismo quanto em atividades de caminhada. O entendimento desses fatores é importante para alertar a população sobre os riscos de realizar tarefas concomitantes ao uso do celular e incentivar os profissionais da saúde a encontrar medidas preventivas para os desequilíbrios e quedas.

A delimitação do público alvo em adultos jovens se deu pela alta prevalência de uso de smartphones nessa faixa etária ${ }^{9}$. Conforme observado na Tabela 1, os participantes utilizam smartphones há mais de dez anos e com uso diário médio de quatro horas. Esse valor, ainda que considerado alto por alguns autores, é inferior à média observada em estudos que abordam jovens com vícios e dependências no uso do celular ${ }^{16,17}$.

Atividades que demandam dupla-tarefa, como é o caso das atividades propostas nesse estudo, exigem um complexo processamento cognitivo ${ }^{18,19}$. A inclusão de testes cognitivos se deu pela intenção dos autores de analisar a cognição dos participantes antes da realização de dupla-tarefa. Os achados aqui encontrados demonstram escores normais tanto para cognição geral quanto para funções executivas pré-frontais ${ }^{20,21}$.

A interferência do uso do smartphone sobre o equilíbrio humano já foi identificada em estudos prévios. No entanto, do nosso conhecimento, esse é o primeiro estudo brasileiro que aborda a temática do equilíbrio no ato concomitante de usar o smartphone. Os resultados encontrados nessa amostra brasileira corroboram os dados publicados em estudos internacionais ${ }^{7,8,22}$.

Sobre o equilíbrio estático, o uso do smartphone afetou as variáveis estabilométricas relacionadas à instabilidade da pessoa, como a área e a velocidade de deslocamento. Resultado surpreendente para os autores foi o fato de que falar ao telefone em pé ocasiona maior desequilíbrio e risco de quedas do que a atividade de escrever uma mensagem. Tendo em vista a alta complexidade cerebral envolvida na escrita ${ }^{23}$, associado à motricidade fina do ato de digitar ao celular. Os autores acreditavam que a atividade de digitação traria mais dificuldades aos participantes do que o ato de falar ao celular. Os resultados encontrados divergiram das hipóteses iniciais dos autores e esses devem servir de incentivo para novos estudos nessa temática. 
O equilíbrio dinâmico, verificado na atividade do TUG, identificou o impacto que o uso do smartphone exerce na realização da caminhada. Nesse caso, não ocorreu diferença de rendimento na comparação das atividades de digitar mensagens com a tarefa de falar ao celular. O grave é que, conforme sugerido por Lin e Huang ${ }^{24}$, a dupla-tarefa do smartphone ao caminhar predispõem as pessoas a maiores riscos e acidentes nas ruas.

A Tabela 4, contendo os tamanhos do efeito da interferência do smartphone sobre o equilíbrio dos participantes, corrobora as tabelas anteriores que detalham as análises dos níveis de significâncias. Isto é, as comparações que apresentaram diferença significativa para a interferência do uso do smartphone sobre o equilíbrio apresentaram tamanho do efeito forte e do poder estatístico acima de $98 \%$. As análises que não apresentaram interferência do uso de smartphone sobre o equilíbrio (deslocamento anterior e médio-lateral) apresentaram tamanho do efeito fraco e com poder estatístico pouco expressivo (abaixo de $32 \%$ ). Esse resultado foi importante, visto que demonstra que os erros estatísticos tipo $1 \mathrm{e}$ tipo 2 foram controlados nesse estudo.

Por fim, os autores optaram por excluir as pessoas que não tinham e aquelas que nunca utilizaram um aparelho smartphone porque é possível que essas pessoas, caso fossem incluídas, tivessem dificuldades no manuseio e no entendimento do aparelho, gerando viés sobre os resultados. De forma similar, os pesquisadores optaram por utilizar o smartphone do próprio participante nos testes para não ocorrer interferência de dificuldades de manuseio de um aparelho novo sobre os resultados.
Limitações

Os resultados dessa pesquisa devem ser analisados diante de algumas limitações. Em primeiro lugar, a análise do tamanho do efeito predisse à necessidade amostral de 45 participantes. No presente estudo os autores avaliaram apenas 10 participantes, quantitativo esse impactado negativamente pela crise de saúde pública ocorrida entre os anos 20192021, que demandou restrições de isolamento social diante da Covid-19. Em segundo lugar, alguns fatores que podem afetar o equilíbrio humano, como a prática de atividade física, a força muscular e a rotina dos participantes não foram incluídos e podem ser possíveis fatores de confusão. Mesmo diante dessas limitações, os achados são importantes e devem incentivar novas pesquisas na temática aqui abordada.

\section{CONCLUSÃO}

Adultos jovens apresentaram desequilíbrio e riscos aumentados de quedas quando realizaram atividades concomitantes ao uso de smartphone. Os resultados são significativos, no entanto, devem ser analisados com cautela diante da sugestão de um tamanho amostral maior.

\section{AGRADECIMENTOS}

Os autores agradecem o suporte financeiro do Conselho Nacional para o Desenvolvimento Científico e Tecnológico (por meio de bolsas de iniciação científica), da Coordenação de Aperfeiçoamento de Pessoal de Nível Superior (por meio de bolsas de mestrado, código 001) 
e dos Programas de Pós-graduação Saúde e

Desenvolvimento e Ciências do Movimento da Universidade Federal de Mato Grosso do

Sul, pelo apoio logístico.

\section{REFERÊNCIAS}

1. Dorsey ER, Yvonne Chan YF, McConnell MV, Shaw SY, Trister AD, Friend SH. The use of smartphones for health research. Acad Med. 2017; 92(2):157-160.

2. Villanti AC, Johnson AL, llakkuvan V, Jacobs MA, Graham $A L$, Rath JM. Social media use and access to digital technology in US young adults in 2016. J Med Internet Res. 2017;19(6):e196.

3. O'Connor S, Andrews T. Smartphones and mobile applications (apps) in clinical nursing education: a student perspective. Nurse Educ Today. 2018; 69:172-178.

4. Thoma B, Turnquist A, Zaver F, Hall AK, Chan TM. Communication, learning and assessment: exploring the dimensions of the digital learning environment. Med Teach. 2019; 41(4):385-390.

5. Takeuchi N, Mori T, Suzukamo Y, Tanaka N, Izumi S. Parallel processing of cognitive and physical demands in left and right prefrontal cortices during smartphone use while walking. BMC Neurosci. 2016;17:9.

6. Beurskens R, Steinberg F, Antoniewicz F, Wolff W, Granacher U. Neural correlates of dual-task walking: effects of cognitive versus motor interference in young Adults. Neural Plast. 2016; 2016:8032180.

7. Crowley P, Madeleine P, Vuillerme N. The effects of mobile phone use on walking: a dual task study. BMC Res Notes. 2019;12(1):352.

8. Hyong $\mathrm{IH}$. The effects on dynamic balance of dual-tasking using smartphone functions. J Phys Ther Sci. 2015; 27(2):527-529.

9. IBGE - Instituto Brasileiro de Geografia e Estatística. Pesquisa Nacional por Amostra de Domicílios Contínua 2017. - Acesso à Internet e à televisão e posse de telefone móvel celular para uso pessoal [acesso em 05 maio 2020].

10. Cuschieri S. The STROBE guidelines. Saudi J Anaesth. 2019;13(Suppl 1):S31-S34.

11. Folstein MF, Folstein SE, McHugh PR. "Mini-mental state": a practical method for grading the cognitive state of patients for the clinician. J Psychiatr Res. 1975; 12(3):189-198.

12. Dubois B, Slachevsky A, Litvan I, Pillon B. The FAB: a Frontal Assessment Battery at bedside. Neurology. 2000;55(11):1621-1626.

13. Lezak MD. Neuropsychological assessment (3rd ed.). New York, NY: Oxford University Press, 1995.
14. Pfeffer RI, Kurosaki TT, Harrah CH Jr, Chance JM, Filos S. Measurement of functional activities in older adults in the community. J Gerontol. 1982; 37(3):323-329.

15. Podsiadlo D, Richardson S. The timed "Up \& Go": a test of basic functional mobility for frail elderly persons. J Am Geriatr Soc. 1991;39(2):142-148.

16. Hanafi E, Siste K, Wiguna T, Kusumadewi I, Nasrun MW Temperament profile and its association with the vulnerability to smartphone addiction of medical students in Indonesia. PLoS One. 2019;14(7):e0212244.

17. Celikkalp U, Bilgic S, Temel M, Varol G. The smartphone addiction levels and the association with communication skills in nursing and medical school students. J Nurs Res. 2020.

18. Bayot M, Dujardin K, Tard C, Defebvre L, Bonnet CT, Allart $E$, Delval A. The interaction between cognition and motor control: A theoretical framework for dual-task interference effects on posture, gait initiation, gait and turning. Neurophysiol Clin. 2018; 48(6):361-375.

19. Lim J, Chang SH, Lee J, Kim K. Effects of smartphone texting on the visual perception and dynamic walking stability. J Exerc Rehabil. 2017;13(1):48-54.

20. Brucki SMD, Nitrini R, Caramelli P, Bertolucci PHF, Okamoto $\mathrm{IH}$. Suggestions for utilization of the mini-mental state examination in Brazil. Arq Neuropsiquiatr. 2003; 61(3B):777-81.

21. Beato RG, Nitrini R, Formigoni AP, Caramelli P. Brazilian version of the Frontal Assessment Batther (FAB): preliminar data on administration to healthy elderly. Dement Neuropsychol. 2007; 1:59-65.

22. Lee $\mathrm{JH}$, Lee $\mathrm{MH}$. The effects of smartphone multitasking on gait and dynamic balance. J Phys Ther Sci. 2018;30(2):293-296.

23. Longcamp M, Richards TL, Velay JL, Berninger VW Neuroanatomy of handwriting and related reading and writing skills in adults and children with and without learning disabilities: French-american connections. Pratiques. 2016;171-172.

24. Lin MB, Huang YP. The impact of walking while using a smartphone on pedestrians' awareness of roadside events. Accid Anal Prev. 2017; 101:87-96.

\section{CORRESPONDÊNCIA}

Gustavo Christofoletti

Av. Costa e Silva s/n, Instituto Integrado de Saúde,

UFMS/Cidade Universitária

Campo Grande/MS, Brasil. CEP: 79070-900.

Email: g.christofoletti@ufms.br 